\title{
Contrast Enhancement of Images Using Meta-Heuristic Algorithm
}

\author{
Sunkavalli Jaya Prakash ${ }^{1 *}$, Manna Sheela Rani Chetty ${ }^{1}$, Jayalakshmi A ${ }^{2}$ \\ ${ }^{1}$ Department of Computer Science and Engineering, Koneru Lakshmaiah Education Foundation, Vaddeswaram 522502, AP, \\ India \\ ${ }^{2}$ Department of Computer Science and Engineering, Prasad V. Potluri Siddhartha Institute of Technology, Kanuru 520007, \\ Vijayawada, AP, India
}

Corresponding Author Email: prakashsunkavalli@sircrrengg.ac.in

https://doi.org/10.18280/ts.380509

Received: 9 August 2021

Accepted: 2 October 2021

\section{Keywords:}

image processing, contrast enhancement, meta-heuristic, chaotic crow search, optimization

\begin{abstract}
One of the most important processes in image processing is image enhancement, which aims to enhance image contrast and quality of information. Due to the lack of adequate conventional image enhancement and the challenge of mean shift, intelligence-based image enhancement systems are becoming an essential requirement in image processing. This paper proposes a new approach for enhancing low contrast images utilizing a modified measure and integrating a new Chaotic Crow Search (CCS) and Krill Herd (KH) Optimization-based metaheuristic algorithm. Crow Search Algorithm is a cutting-edge meta-heuristic optimization technique. Chaotic maps are incorporated into the Crow Search Method in this work to improve its global optimization. The new Chaotic Crow Search Algorithm depends on chaotic sequences to replace a random location in the search space and the crow's recognition factor. Based on a new fitness function, Krill Herd optimization is utilized to optimize the tunable parameter. The fitness function requires different primary objective functions that use the image's edge, entropy, grey level co-occurrence matrix (GLCM) contrast, and GLCM energy for increased visual, contrast, and other descriptive information. The results proved that the suggested approach outperforms all-new methods in terms of contrast, edge details, and structural similarity, both subjectively and statistically.
\end{abstract}

\section{INTRODUCTION}

Contrast enhancement is among the most crucial stages of image enhancement. It could be used to increase the range of intensities in a grey-scale image, disclose portions of the image that are hard to find, or transform the histogram of images so that they are easier to comprehend by persons. Contrast enhancement is a typical preprocessing step in most digital image processing, to enhance the visual impression of an image [1]. Image enhancement can make it easier for a human observer to comprehend images as well as for machines to recognize them [2]. As a result, it is widely used in a variety of disciplines, including "Medical Image Analysis", "Human Vision", "Defect Detection", and "Remote Sensing" [3]. There may be limits in the image technologies and also atmospheric interferences that reduce the image quality. Currently, a majority of new and multilateral enhancement-based methods are being used to improve picture quality to the point where the image can be identified without losing information. To name a few, tonal changes, clarity, luminance, and contrast correction are all aspects of image enhancement that should be considered. For contrast enhancement, the traditional approach of histogram equalization is typically utilized [4]. This method has the severe disadvantage of causing data loss and giving the image a false aspect by over-enhancing it [5]. To solve the disadvantages of the histogram equalization approach, many histogram modification strategies have been developed. A strategy for improving the IR picture via histogram equalization has been presented [6]. To separate the background and foreground sections of the histogram, a threshold method needs. The threshold limits are improved by meta-heuristics optimization to minimize the overenhancement of the image.

One of the most fundamental approaches for image pixels improvement of gray-level images is histogram transformation [7]. The fundamental connection of the pixel's intensity values would be altered when the histogram is adjusted. The word histogram corresponds to a finite probability distribution function for gray levels that measures the average descriptive statistics of the gray levels. Histogram equalization is a contrast adjustment approach in image processing that helps in evaluating the image's global contrast, especially when the image's relevant data is processed by near contrast values [8]. However, pixel intensity rates will be modified to an average location, which will conceal the original image's features. It generally adjusts the histogram via the transformation function to have the desired goal, which is fundamental for pixel-level domain approaches of image contrast enhancement. As a result, this technique delivers the most information possible from the original image. Linear contrast stretching extends the image quality of the gray levels in the source image by applying a linear adjustment. It mostly improves the initial image's contrast level, although the threshold should be manually set. If the threshold isn't high enough, the image enhancement will be poorer than the source. Due to the lack of a common image quality standard that might be used as a design requirement for image enhancement techniques, increasing image contrast by just extending the image's histogram or applying basic gray- 
level modifications is challenging [9].

To overcome the challenge of image enhancement, various innovative approaches based on evolutionary computing and metaheuristic algorithms based on some performance criteria of image enhancement have recently been proposed. The hybridization of meta-heuristics has now gained a lot of interest. We utilize contrast enhancement in the sense of greyscale image intensity modification in this paper. The transfer function, that converts input to output intensities, comes in a variety of forms. As a result, meta-heuristics are particularly suitable for optimization in this enormous search space.

To get better outcomes, we propose a meta-heuristics approach that includes Chaotic Crow Search and Krill Herd Optimization methods. First, we looked into the "Krill herd (KH)" Optimization method using certain parameter parameters. It's a swarm intelligence technique that's inspired by a krill individual's herding behavior. Second, for the identical objective, we evaluated the "Chaotic Crow Search (CCS) method". The integration of chaotic theory into the cuckoo search technique results in a new CCS optimization technique proposed in this work. CCS combines chaotic features with CS to improve its performance.

The remainder of the paper is structured out as follows: Section 2 provides a summary of the existing research. Section 3 involves a systematic overview of the proposed "Chaotic Crow Search (CCS) and Krill Herd (KH) Optimization" approach. Section 4 introduces the experimental findings and discussions of the proposed CCS and KH technique. Section 5 concludes with an overview of the proposed work.

\section{RELATED WORK}

There have been several studies on image enhancement that have been published. The primary categories of these techniques include vector functions, structural systems, transform operations, and pseudo texture processes [10]. Point operation employs techniques such as image segmentation, frame filtering, and histogram modeling [10]. Because these are the most basic and common techniques for image enhancement, they are frequently used in research. However, because they have a higher global influence than a local effect, they suffer from image contrast stretching. To enhance the entropy, indirect systems mainly modify the image histogram. Spatial procedures, on the other hand, define contrast measurement criteria and improve the image by doing so [11]. Based on the fuzzy entropy concept and fuzzy set theory, fuzzy logic was implemented as an adaptive direct evaluation technique [11]. The suggested approach outperformed the Adaptive Contrast Enhancement approach, according to the authors; nevertheless, the model is evaluated objectively.

Munteanu and Rosa [10] presented a contrast enhancement transformation technique and implemented the Evolutionary Algorithm as a global search method for the optimal enhancement in 2004. The authors were also to apply a heuristic approach for image enhancement, and they evaluated their methodology using both qualitative and quantitative approaches, highlighting its performance over Linear Stretching and Histogram Equalization. Russo [12] presented an objective measurement approach based on edge gradient histograms in 2005. Rosso's approach creates overshoots along with the image outlines while outperforming both classification and regression un-sharp screening techniques.
Particle Swarm Optimization (PSO) was suggested by Kwok et al. [13] in 2006 as an intensity-preserving method for contrast enhancement. To acquire suitable gamma ratios for the enhancement procedure, the PSO approach will be applied. The use of mean intensity as an objective measure of evaluation is inadequate for making decisions, necessitating the inclusion of wide metrics.

PSO was implemented by Gorai and Ghosh [14] to optimize the desire to advance an augmented image created with Munteanu's algorithms [10]. PSO outperformed other methods, according to Gorai and Ghosh [14]. Ghosh et al. [15] looked at the usage of the Cuckoo Search (CS) technique for image enhancement. In comparison to PSO, Genetic Algorithm, Linear Contrast Stretching, and Histogram Equalization, it was shown that CS performs better. Furthermore, several research have suggested analyzing GIs, with the majority being that meta-heuristics approaches give superior image enhancement when transformation functions are used [16]. Moreover, standardized evaluations for evaluating the efficiency of these techniques are frequently lacking.

Guha et al. [17] developed a chaotic-based whale survival technique that uses a wrapper approach for classification and a filter-based methodology for selecting a subset of characteristics. They have utilized four different types of chaotic maps, including circular, logistical, piecewise, and tent, to influence whales' movement patterns when they're on the hunt. To address optimization issues, Arora et al. [18] used a hybrid-based method that combined the Crow Search Algorithm with Grey Wolf Optimization [19]. Ahmed and Ali [20] introduced a hybrid-based optimization approach for finding the best features in medical analytical techniques. They combined chaos theory with the crow search algorithm and the fuzzy c-means method. The CSA is used to accomplish global optimization and reduce the sensitivity of local optimization. The FCM object method is applied as a cost function for the chaotic CSA.As a result, we offered new quantitative metrics to evaluate an algorithm's performance by examining both $\mathrm{KH}$ and $\mathrm{CS}$ in our paper.

\section{PROPOSED SYSTEM}

The proposed system uses meta-heuristics such as Cuckoo Search and Krill-Herd Optimization to enhance the contrast of the input images. The suggested technique is illustrated in Figure 1. The collected sample images are preprocessed by a "Gamma-Corrected Histogram Equalization" that is improved using a meta-heuristics optimization approach to enhance image contrast.

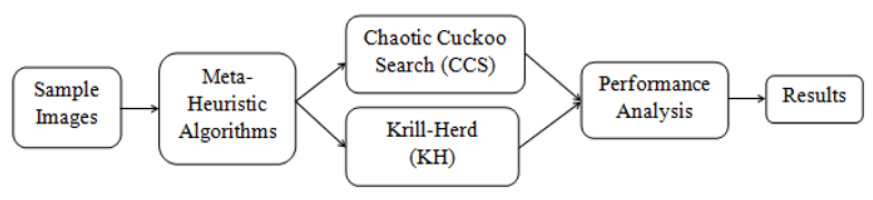

Figure 1. Proposed system

One of the most fundamental processes in image enhancement is contrast enhancement. Enhancing the contrast is among the key phases in image enhancement. It includes enhancing the image's intensity values or manipulating the image histogram such that people can understand it. Global intensity transformation is the most widely applied contrast enhancement technique. The input intensities are mapped to a 
unique level of intensity rates using a transformation function. The contrast enhancement approach applied in this study is illustrated in Figure 2, which is the system architecture.

The input image is subjected to global histogram equalization. Because it reduces the image's contrast ratio, a minor change of histogram equalization is ineffective for image processing. A sample image used in a system like an image fusion must maintain as much data as possible from the input images and be usable for even more evaluation. To achieve this, the medium contrast images were further enhanced using an improved gamma correction approach.

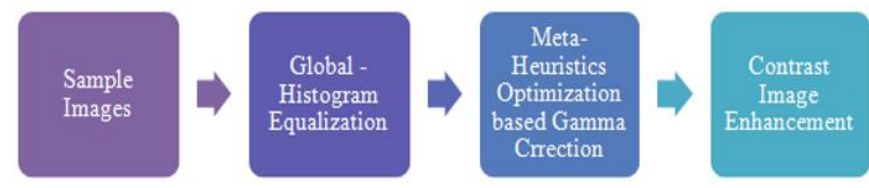

Figure 2. Schematic diagram of the contrast enhancement approach

\subsection{Global histogram equalization}

"Global Histogram (GH) Equalization" is a technique for improving an image's dynamic range. It changes the intensities in the source images to create a homogeneous spatial feature in the output image.

Let $G=\{G(a, b)\}$ be the input image, with $G(a, b)$ denoting the image's intensity difference, G.i denotes the total no. of pixels in $\mathrm{G}$, and the intensities in $\mathrm{G}$ are classified into $\mathrm{Z}$ dimensions: $G_{0}, G_{1}, G_{2}, \ldots, G_{z}[6]$.

Eq. (1) may be used to calculate the "Probability Density Function (PDF)" of an image intensity level $G_{m}$ :

$$
D\left(G_{m}\right)=\frac{i_{m}}{i}, m=0,1,2, \ldots, z-1
$$

The total no. of pixels with the specified intensity, $G_{m}$ is represented by $i_{m}$.

The "cumulative distribution function (CDF)" may be derived from Eq. (2), based on the PDF.

$$
E\left(G_{m}\right)=\sum_{f=1}^{m} \frac{i_{m}}{i}, m=0,1,2, \ldots, z-1
$$

It is a well-known observation of Eq. (2): $\sum_{m=0}^{z-1} \mathrm{E}\left(G_{m}\right)=1$. The CDF, which is given in Eq. (3), is now used to build a transfer function $\mathrm{R}(\mathrm{a})$.

$$
R\left(G_{m}\right)=G_{0}+\left(G_{z-1}-G_{0}\right) \cdot \mathrm{E}\left(G_{m}\right)
$$

Now, Eq. (4) is used to construct the output image U, which is the enhanced image:

$$
U=R(G)=\left\{R\left(G(a, b) \mid \forall G(a, b) \in\left\{G_{0}, G_{1}, G_{2}, \ldots \ldots G_{z-1}\right\}\right)\right\}
$$

The limitation of this approach is that it is unable to preserve the output image's mean luminance. To do this, certain brightness variations may be made to get it to the ideal levels. Gamma correction is used to do this.

\subsection{Gamma correction}

The "Gamma Correction" is an image and localized exponentially correcting method for improving image contrast. It's a low-cost method that works well with both dark and light images. On the other hand, individually selecting the gamma value depends on the image might be time-consuming, particularly when dealing with input images [21].

The resulting image is generated using Eq. (5) for a conventional gamma correction.

$$
G_{o u t}=E \cdot G_{i n}^{\alpha}
$$

The intensities of the input and output images are represented by $G_{\text {in }}$ and $G_{\text {out }}$, accordingly. The curvature of the transforming slope is determined by the values of $E$ and $\alpha$. The rate of $\mathrm{c}$ changes relating to the qualities of the image.

Compression of levels of intensity towards darker levels while concurrently extending intensities toward brighter ones is an alternative approach for contrast enhancement.

The transformation function may be expressed as Eq. (6) for images with low and medium contrast:

$$
G_{\text {out }}=\beta G_{E D}+(1+\beta) G_{\text {sa }}
$$

The original image is compressed to darker levels in $G_{E D}$, while the source image is extended to brighter ones in $G_{s a}$.

Eq. (7) is used to calculate $G_{E D}$ :

$$
G_{E D}=G_{i n}^{\alpha}
$$

Eq. (8) is used to calculate $G_{s a}$ :

$$
G_{s a}=1-\left(1-G_{i n}\right)^{\beta}
$$

The histogram-equalized image is represented by $G_{i n}$.

It is obvious from Eqns. (6)-(8) that the contrast-enhanced image is determined by two factors, $\alpha$ and $\beta$. The factors $\alpha$ and $\beta$, must be adjusted based on pictures [22] to guarantee that the augmentation is within specified limit with no color changes.

The parameters to be controlled in the transformation function are $\alpha$ and $\beta$. As a result, the parameters to be improved are $\alpha$ and $\beta$. The control parameters are optimized using metaheuristic approaches such as Chaotic Crowf Search (CCS) and Krill-Herd (KH) based optimization.

\subsection{Chaotic crow search (CCS) algorithm}

Askarzadeh [23] introduced the CSA algorithm in 2016. The basic source of support for this program was a crow's food-hiding technique. Crows are said to be among the finest intelligent birds on the globe. They have a bigger brain than their physical size suggests. Crows are smart creatures. Their brains are somewhat smaller than human brains. In contrast, they are identified in the mirror test. They can understand faces. When they find one hostile crow, they use complex communication to alert the other crows. They can also keep and recollect their meals for a few decades. They have a reputation for taking the food of other birds; hence they are known to be thieves. They utilize their previous experience as a thief to predict the conduct of a pilferer. They're suspicious. They change their hiding locations when a crow reports a theft. As a result, they can eliminate any potential victims in the future.

The term "chaos" refers to the phenomena. Any change in the original state may result in non-linear behavior in the future. One of the most current search methods is chaos optimization. The basic idea is to transform chaotic criteria into optimum solutions. Strategic decisions, homogeneity, and 
stochasticity are all characteristics of chaotic action that are used to find a global solution. COA's primary advantages are its quick convergence rate and desire to prevent local minima. All of these benefits may effectively enhance the quality of evolutionary computation [24]. "Chaotic maps" are definite in the view that they don't include any random components. To generate chaotic sets, eleven well-known non-invertible onedimensional mappings are used.

The random variables used to determine the crow movements are substituted with chaotic factors in this section. The usage of a chaotic sequence created from chaotic maps is applied since updating the crow position affects the optimal solution and convergence rate. CCSA is characterized as a mixture of chaos and CCSA. The optimization technique in this work contains 10 various chaotic maps. A unique binary CCSA for the feature selection problem is demonstrated in this paper. The solution pool in binary CCSA is in binary form, with solutions limited to binary 0 and 1 .

In CCSA's searching iterations, a chaotic sequence is integrated. CCSA is used to choose the best feature subset for characterizing the dataset. The goal of feature selection is to enhance classification results, minimize the length of feature subsets, and lower the computational cost. The processing is completed when the optimization approach reaches the convergence rate or when the optimum solution can be found. We utilized the maximum number of iterations in our instance. Algorithm 1 defines the CCSA pseudo-code and also the flowchart [25] that corresponds to it.

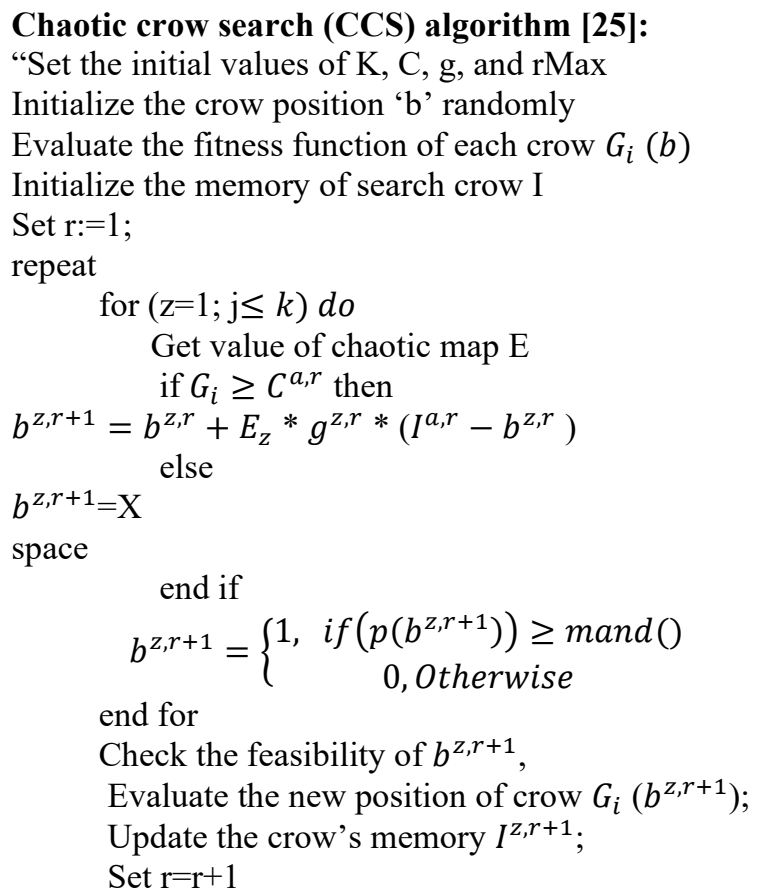
until $(\mathrm{r}<\mathrm{rMax})$

Produce the best solution I."

\subsection{Proposed krill-herd (KH) optimization}

The 'krill herd (KH)' optimization method is used in the proposed model to maximize or minimize the fitness function to produce an optimal value for the adjustable parameter [26]. For continuous optimization problems, the $\mathrm{KH}$ method is a swarm intelligence technique [27]. It has been established to outperform or be equivalent to several existing algorithmic approaches. When compared to other swarm-intelligence techniques, it is simple to build, resilient, and involves several control parameters, with just one parameter requiring finetuning [27].

In the KH algorithm, krill's hunt for food in a multidimensional search space, with individual krill locations defined as decision variables and the distance between the krill's and the best resource corresponding to the magnitude of the objective value. The three operational mechanisms that determine a krill's time-dependent position are as follows:

"Motion-Induced Processes, Foraging Movement, and Random Physical Diffusion" are all examples of motioninduced processes. The following sections give descriptions and mathematical expressions of various operating processes:

\subsubsection{Motion induced process}

Individual krill are driven toward high density by mutual effects, which are represented by Eq. (9). The target swarm, local swarm, and repulsive swarm densities are used to determine the direction in which krill individuals $\left(\beta_{z}\right)$ can learn.

$$
\begin{gathered}
K_{z}^{\text {new }}=K^{\text {max }} \beta_{z}+\gamma_{i} K_{z}^{\text {old }} \\
\beta_{z}=\beta_{z}^{\text {local }}+\beta_{z}^{\text {target }}
\end{gathered}
$$

where, $K^{\max }, \gamma_{i}$, and $K_{z}^{\text {old }}$ represent the maximum induced motion, inertia weight, and latest induced speed, correspondingly. For the optimal krill individual, $\beta_{z}$ is combined with the local influence $\left(\beta_{z}^{\text {local }}\right)$ due to neighbors and the target direction effect $\left(\beta_{z}^{\text {target }}\right)$. The attractive or repelling influence of krill neighbors is assessed for a local search, and krill migration is characterized as:

$$
\beta_{z}^{\text {local }}=\sum_{x=1}^{G} \hat{E}_{z, x} \hat{B}_{z, x}
$$

where, $\hat{E}_{z, x}$ signifies the corresponding location of $\hat{E}_{z, x}$ and $\widehat{B}_{z, x}$ is the fitness value of the $\mathrm{z}^{\text {th }}$ krill individual for $\mathrm{x}^{\text {th }}(x=1$, $2, \ldots, K)$ neighbour. The global optima are found when the best fitness on the $\mathrm{j}^{\text {th }}$ individual krill is evaluated and represented as:

$$
\beta_{z}^{\text {target }}=H^{\text {best }} \widehat{E}_{z, \text { best }} \widehat{B}_{z, \text { best }}
$$

where, $H^{\text {best }}$ is the functional factor for the finest krill individuals.

\subsubsection{Foraging motion}

The "Foraging Motion" of the $\mathrm{z}^{\text {th }}$ krill individual is characterized by two factors: resource location and prior experience of the resource location. The following is an example of a foraging motion:

$$
\begin{gathered}
D_{z}=U_{g} \partial_{z}+\delta_{g} D_{z}^{\text {old }} \\
\partial_{z}=\partial_{Z}^{\text {resource }}+\partial_{z}^{\text {best }}
\end{gathered}
$$

where, $\delta_{g}$ is the foraging speed's inertia weight and $U_{g}$ is the foraging speed. The best aim of the $z^{\text {th }}$ krill is indicated as $\partial_{z}^{\text {best }}$, while food attractive $\left(\partial_{z}^{\text {resource }}\right)$ is provided to perhaps drive the krill swarm to the global optima.

\subsubsection{Physical diffusion}

For krill individuals, it is a chaotic system in which mobility is represented in the form of a random directional vector $(\omega)$ 
and a maximum diffusion speed $\left(H^{\max }\right)$. The krill's ideal location is indicated by reduced chaotic movements.

$$
H_{z}=H^{\max }+\omega
$$

Local and global systems combine in this optimization approach, producing significant and efficient outcomes. It can also create global minima for any form of restriction with great precision. The best performance of the fitness function is obtained by lowering the lowest sign of the fitness function. There is no need for derivative information since it utilizes a probabilistic search strategy instead of a gradient approach to get the optimum result. Tuning parameters is critical when using meta-heuristic optimization approaches to get the optimal solution. The $\mathrm{KH}$ method employs just a fine-tuned time interval $\left(H_{r}\right)$, one configurable factor of the $\mathrm{KH}$ method, which gives it a significant edge over other nature-inspired algorithms.

\section{EXPERIMENTAL RESULTS}

Quantitative approaches are used to get statistics on image datasets to illustrate the efficiency of the given strategy. The statistical measures use of benchmark datasets from a variety of fields, such as medical, industry, etc. The datasets were from the "UCI Machine Learning Repository" [28]. A few other instances in the adopted datasets have blank values. All of these missing data are substituted in this work by the median of all known values for a feature-provided class. Eq. (16) defines the median technique mathematically. The missing value for a given class $\mathrm{z}^{\text {th }}$ feature is $f_{x, z}$.

The most often occurring result for a feature provided class is changed with the missing value when categorical data are unavailable.

$$
\bar{f}_{x, z}=\operatorname{median}_{x: f_{x, z \in Q m}} f_{x, z}
$$

Distinct statistical measures are used in this section. The "Worst, Best, and Mean fitness values, and Standard Deviation (SD)", the value from 'Wilcoxon's rank-sum test' is used to calculate these principles. "Wilcoxon's rank-sum test" [29] is a non-parametric statistical method with a $5 \%$ threshold of significance. To show that the proposed algorithm is considerably superior to previous techniques, an empirical analysis is required [30]. "Wilcoxon's rank-sum test" is more accurate than the t-test since it assumes linearity of separation between different pair values. Furthermore, because it does not require normal distributions, it is safer than the t-test. Outliers also have less impact on the Wilcoxon test than the t-test [30]. In typically, the highest $P$ rates are those with a $P$ value of less than 0.1 . As a result, it might be regarded as adequate evidence for rejecting the null hypothesis. In this study, this method was used to evaluate the efficiency of every chaotic map and choosing the appropriate model. The following are the quantitative definitions for the "Worst, Best, Mean Fitness Values, and SD":

$$
\begin{gathered}
\mathrm{SD} \sqrt{\frac{\sum_{x=1}^{G}\left(Y F_{x}-\sigma\right)^{2}}{r \operatorname{Max}}} \\
\text { Best }=\max _{x=1}^{r \operatorname{Max} Y F_{x}} \\
\text { Worst }=\min _{x=1}^{\text {rMax } Y F_{x}}
\end{gathered}
$$

$$
\text { Mean }=\frac{1}{r \operatorname{Max}} \sum_{x=1}^{r \operatorname{Max}} \max _{x=1}^{r \operatorname{Max}} Y F_{x}
$$

Results of experiments on the benchmark datasets are carried out in this section. Figure 3 illustrates the statistical results of the proposed work. The purpose of these tests is to assess the proposed CCS and KS Optimization algorithm's performance and compare it to that of existing meta-heuristic methods. All of these tests are run on the same system with the same specifications. The main objective of this experiment is to compare the efficiency of the CCS and $\mathrm{KH}$ techniques with various chaotic maps to find the best chaotic map. The performance of CCS and $\mathrm{KH}$ with a sine chaotic map is evaluated to that of other optimization methods in this section. For addressing the curse of dimensionality, several models have been implemented in the literature. "Particle Swarm Optimization (PSO), Artificial Bee Colony (ABC), and Grey Wolf Optimizer (GWO)" are the concern methods. Figure 4 shows the Comparison of the performance rate of the metaheuristics algorithm.

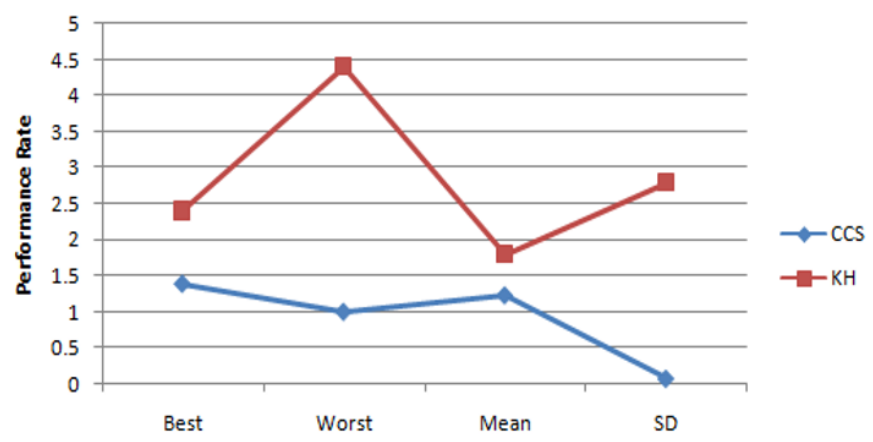

Figure 3. Comparison of statistical results performance

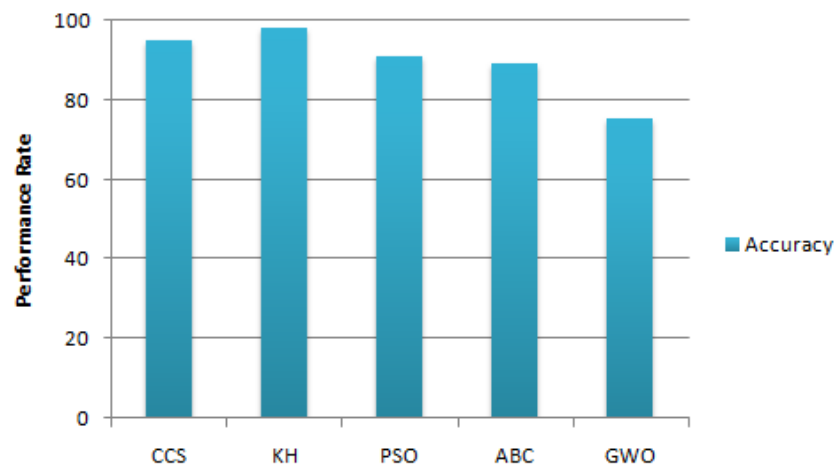

Figure 4. Comparison of performance analysis of metaheuristics algorithm

The major advantage of $\mathrm{CS}$ and $\mathrm{KH}$ is that there are fewer characteristics to configure. Any optimization method's efficiency is greatly affected by these factors. Determining the best value for each, on the other hand, is a challenging process. In this paper, the discrete factors are modified with values derived from the chaotic map, introducing a novel method for identifying the optimum cost function via chaotic maps. The findings of the experiments suggest that chaotic maps, particularly the sine chaotic map, may improve the reliability and efficiency of $\mathrm{CS}$ and $\mathrm{KH}$ optimization techniques. The average score for benchmark dataset iterations is compared in Table 1. As can be observed, CCSA, KH, PSO, ABC, and GWO all have equivalent results.

Without optimization, contrast-enhanced images may also 
result in a dark or overly illuminated image, and certain images, if used in the subsequent fusion stage, will result in a fused image that will not convey the information given in the specific input image, resulting in a poor-quality output image. By optimizing and, which are used as components of the scaling factor in the meta-heuristic approach, sample images with great contrast enhancement could be created without manually altering the control performance, which is highly time-consuming for these sorts of large images.

Table 1. Performance results of meta-heuristics algorithm

\begin{tabular}{cccccc}
\hline Statistics Classifiers & CCS & KH & PSO & ABC & GWO \\
\hline Best & 1.34 & 1.51 & 1.28 & 1.17 & 1.35 \\
Worst & 1.26 & 1.29 & 1.19 & 1.16 & 1.2 \\
Mean & 1.31 & 1.45 & 1.24 & 1.16 & 1.28 \\
SD & 0.05 & 0.09 & 0.03 & 0.01 & 0.03 \\
\hline
\end{tabular}

\section{CONCLUSION}

A novel meta-heuristics technique to enhancing image contrast was proposed in this work. The suggested CCS and $\mathrm{KH}$ optimization methods are innovative approaches to image global change. The proposed optimization methodology aids the new method's automation. The fitness criterion selected for this paper is an enhanced criterion that yields good outcomes. It gives the processed images a more natural appearance. We compared the novel technique against several frequently used strategies, including PSO, ABC, and GWO. According to the results, the new approach outperforms the aforementioned strategies both subjectively and objectively. We also discovered that the suggested technique outperforms the other techniques in terms of uniformity, contrast, mean, best, Worst, and SD scores. The novel approach may be used to enhance images from a human observer's factor of perspective or as a pre-processing task for other image processing applications.

\section{REFERENCES}

[1] Hoseini, P., Shayesteh, M.G. (2013). Efficient contrast enhancement of images using hybrid ant colony optimization, genetic algorithm, and simulated annealing. Digital Signal Processing, 23(3): 879-893. https://doi.org/10.1016/j.dsp.2012.12.011

[2] Pashaei, E., Pashaei, E., Aydin, N. (2020). Hybrid krill herd algorithm with particle swarm optimization for image enhancement. International Conference on Intelligent and Fuzzy Systems, Istanbul, Turkey, pp. 1431-1439. https://doi.org/10.1007/978-3-030-511562_166

[3] Gogna, A., Tayal, A. (2012). Comparative analysis of evolutionary algorithms for image enhancement. International Journal of Metaheuristics, 2(1): 80-100.

[4] Asokan, A., Anitha, J. (2020). Artificial bee colonyoptimized contrast enhancement for satellite image fusion. Artificial Intelligence Techniques for Satellite Image Analysis, pp. 83-105. https://doi.org/10.1007/9783-030-24178-0_5

[5] Wang, X., Chen, L. (2017). An effective histogram modification scheme for image contrast enhancement. Signal Processing: Image Communication, 58: 187-198. https://doi.org/10.1016/j.image.2017.07.009

[6] Wan, M., Gu, G., Qian, W., Ren, K., Chen, Q., Maldague,
X. (2018). Particle swarm optimization-based local entropy weighted histogram equalization for infrared image enhancement. Infrared Physics \& Technology, 91: 164-181. https://doi.org/10.1016/j.infrared.2018.04.003

[7] Ye, Z., Wang, M., Hu, Z., Liu, W. (2015). An adaptive image enhancement technique by combining cuckoo search and particle swarm optimization algorithm. Computational Intelligence and Neuroscience, 2015: 825398. https://doi.org/10.1155/2015/825398

[8] Pizer, S.M., Amburn, E.P., Austin, J.D., Cromartie, R., Geselowitz, A., Greer, T., Romeny, B.H., Zimmerman, J.B., Zuiderveld, K. (1987). Adaptive histogram equalization and its variations. Computer Vision, Graphics, and Image Processing, 39(3): 355-368. https://doi.org/10.1016/S0734-189X(87)80186-X

[9] Hasikin, K., Isa, N.A.M. (2014). Adaptive fuzzy contrast factor enhancement technique for low contrast and nonuniform illumination images. Signal, Image and Video Processing, 8(8): 1591-1603. https://doi.org/10.1007/s11760-012-0398-x

[10] Munteanu, C., Rosa, A. (2004). Gray-scale image enhancement as an automatic process driven by evolution. IEEE Transactions on Systems, Man, and Cybernetics, Part B (Cybernetics), 34(2): 1292-1298. https://doi.org/10.1109/TSMCB.2003.818533

[11] Cheng, H.D., Min, R., Zhang, M. (2010). Automatic wavelet base selection and its application to contrast enhancement. Signal Processing, 90(4): 1279-1289. https://doi.org/10.1016/j.sigpro.2009.10.013

[12] Russo, F. (2005). Automatic enhancement of noisy images using objective evaluation of image quality. IEEE Transactions on Instrumentation and Measurement, 54(4): $1600-1606$ https://doi.org/10.1109/TIM.2005.851084

[13] Kwok, N.M., Ha, Q.P., Liu, D.K., Fang, G. (2006). Intensity-preserving contrast enhancement for gray-level images using multi-objective particle swarm optimization. In 2006 IEEE International Conference on Automation Science and Engineering, Shanghai, China, pp. 21-26. https://doi.org/10.1109/COASE.2006.326849

[14] Gorai, A., Ghosh, A. (2009). Gray-level image enhancement by particle swarm optimization. 2009 World Congress on Nature \& Biologically Inspired Computing (NaBIC), Coimbatore, India, pp. 72-77. https://doi.org/10.1109/NABIC.2009.5393603

[15] Ghosh, S., Roy, S., Kumar, U., Mallick, A. (2014). Gray level image enhancement using cuckoo search algorithm. Advances in Signal Processing and Intelligent Recognition Systems, pp. 275-286. https://doi.org/10.1007/978-3-319-04960-1_25

[16] Maragatham, G., Roomi, S.M.M. (2015). A review of image contrast enhancement methods and techniques. Research Journal of Applied Sciences, Engineering and Technology, 9(5): 309-326. https://doi.org/10.19026/rjaset.9.1409

[17] Guha, R., Ghosh, M., Mutsuddi, S., Sarkar, R., Mirjalili, S. (2020). Embedded chaotic whale survival algorithm for filter - wrapper feature selection. Soft Computing, 24(17): 12821-12843. https://doi.org/10.1007/s00500020-05183-1

[18] Arora, S., Singh, H., Sharma, M., Sharma, S., Anand, P. (2019). A new hybrid algorithm based on grey wolf optimization and crow search algorithm for unconstrained function optimization and feature 
selection. IEEE Access, 7: 26343-26361. https://doi.org/10.1109/ACCESS.2019.2897325

[19] Reenadevi, R., Sathiya, T., Sathiyabhama, B. (2021). Classification of digital mammogram images using wrapper based chaotic crow search optimization algorithm. Annals of the Romanian Society for Cell Biology, 25(5): 2970-2979.

[20] Anter, A.M., Ali, M. (2020). Feature selection strategy based on hybrid crow search optimization algorithm integrated with chaos theory and fuzzy c-means algorithm for medical diagnosis problems. Soft Computing, 24(3): 1565-1584. https://doi.org/10.1007/s00500-019-03988-3

[21] Rahman, S., Rahman, M.M., Abdullah-Al-Wadud, M., Al-Quaderi, G.D., Shoyaib, M. (2016). An adaptive gamma correction for image enhancement. EURASIP Journal on Image and Video Processing, 2016: 35. https://doi.org/10.1186/s13640-016-0138-1

[22] Jiang, G., Wong, C.Y., Lin, S.C.F., Rahman, M.A., Ren, T.R., Kwok, N., Shi, H., Yu, Y.H., Wu, T. (2015). Image contrast enhancement with brightness preservation using an optimal gamma correction and weighted sum approach. Journal of Modern Optics, 62(7): 536-547. https://doi.org/10.1080/09500340.2014.991358

[23] Askarzadeh, A. (2016). A novel metaheuristic method for solving constrained engineering optimization problems: Crow search algorithm. Computers \& Structures, 169: 1-12. https://doi.org/10.1016/j.compstruc.2016.03.001

[24] Zhang, Q., Li, Z., Zhou, C.J., Wei, X.P. (2013). Bayesian network structure learning based on the chaotic particle swarm optimization algorithm. Genetics and Molecular Research, $\quad 12(4)$ : 4468-4479. https://doi.org/10.4238/2013.october. 10.12

[25] Sayed, G.I., Hassanien, A.E., Azar, A.T. (2019). Feature selection via a novel chaotic crow search algorithm. Neural Computing and Applications, 31(1): 171-188. https://doi.org/10.1007/s00521-017-2988-6

[26] Kandhway, P., Bhandari, A.K., Singh, A. (2020). A novel reformed histogram equalization based medical image contrast enhancement using krill herd optimization. Biomedical Signal Processing and Control, 56: 101677. https://doi.org/10.1016/j.bspc.2019.101677

[27] Bolaji, A.L.A., Al-Betar, M.A., Awadallah, M.A., Khader, A.T., Abualigah, L.M. (2016). A comprehensive review: Krill Herd algorithm $(\mathrm{KH})$ and its applications. Applied Soft Computing, 49: 437-446. https://doi.org/10.1016/j.asoc.2016.08.041

[28] Bache, K., Lichman, M. UCI Machine learning repository. http://archive.ics.uci.edu/ml, accessed on July 19, 2016.

[29] Wilcoxon, F. (1992). Individual comparisons by ranking methods. Breakthroughs in Statistics, pp. 196-202. https://doi.org/10.1007/978-1-4612-4380-9_16

[30] Derrac, J., García, S., Molina, D., Herrera, F. (2011). A practical tutorial on the use of nonparametric statistical tests as a methodology for comparing evolutionary and swarm intelligence algorithms. Swarm and Evolutionary Computation, $1(1)$ : 3-18. 